\title{
Haptic Intention Augmentation for Cooperative Teleoperation
}

\author{
Michael Panzirsch, Ribin Balachandran, Jordi Artigas, Cornelia Riecke, Manuel Ferre, Alin Albu-Schaeffer
}

\begin{abstract}
Multiple robotic agents, autonomous or teleoperated, can be employed to synergise and cooperate to achieve a common objective more effectively. Tasks using robotic manipulators can be eased and improved in terms of reliability, adaptability and ergonomics via robot cooperation. In spite of visual and haptic feedback, cooperative telemanipulation of multiple robots by distant operators can still be challenging due to practical limitations in synchronisation and supervision. This paper presents a new control approach for haptic intention augmentation between two human operators handling objects via teleoperation in a cooperative manner. The force feedback to each operator is enhanced by information on the motion intention of the other operator observed by a force sensor at the input devices. Besides on-ground experiments, an experiment is presented that involves the cooperative teleoperation of an on-ground robot by a cosmonaut on the International Space Station and another distant operator on ground. Index Terms - teleoperation, haptic augmentation, operator intention, cooperation, ISS, time delay
\end{abstract}

\section{INTRODUCTION}

Progress in robotic technologies such as the development of faster processors, lighter actuators and smarter sensors, has paved the path for robots to enter the service sector now more than ever before. Especially in harmful areas like space, nuclear power plants and oil platforms, service robots are in ever increasing demands. If it is the radioactive nature in nuclear plants and the inaccessible pipes in oil platforms, in space the motivation for the use of robots ([1], [2]) is raised by the high costs of extra vehicular activities (EVA) and the related risks for the cosmonauts.

Teleoperation of robots enables extending human dexterity and cognition to remote and harsh environments. Still, for several tasks, the standard bilateral teleoperation of a single robot provides insufficient capabilities. Especially complex tasks require cooperative robots that can be teleoperated by two human operators. For instance, manipulation tasks that require high dexterity, improved handling capability and increased loading capacity favor multi-operator-multi-robot systems (MOMR, [3]). Other MOMR scenarios may require a second operator with a different viewpoint. Also, a local supervising operator may need support by a distant expert operator in a MOMR system.

Cooperation among space robots e.g. has been studied in several works. In [4] the history and the worldwide planned

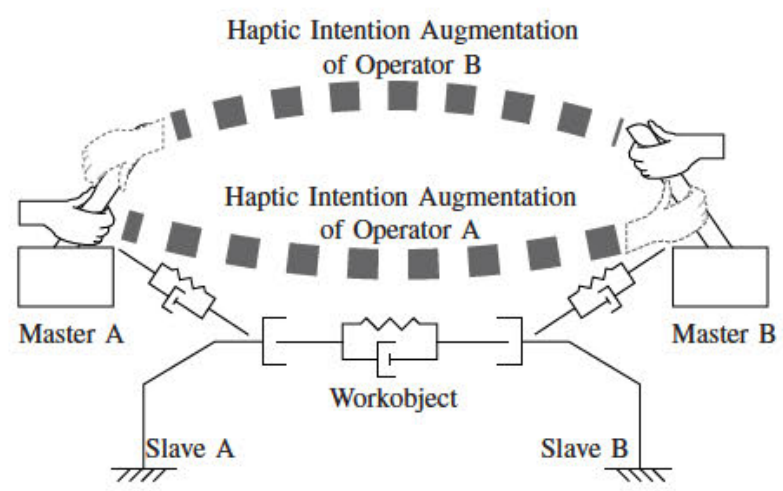

Fig. 1. Functionality of the Haptic Intention Operation

missions for space robot cooperation was reviewed. The analysis yielded that multi-robot systems have advantages in terms of weight reduction and increase of robustness via redundancy. [5] focuses the effects of time delay in MOMR. Different control methods as predictive displays were proposed to prevent collisions caused by the delay. In [6] and [7] i.a. NASA analyzed robot cooperation from the human-robot interaction perspective.

On ground, several experiments have validated the concept of multi-robot teleoperation. The application of modelmediated teleoperation was analyzed in [8]. The authors of [9] and [3] developed a robust control design based on the $\mu$-synthesis and nonlinear adaptive controllers for MOMR systems respectively. In [10], a mobile puma robot was controlled with two joysticks by two distant operators. In [11], an event-based distributed controller for an MOMR system was developed with a mobile robot and a robotic arm with real-time force feedback on two joysticks. In the present work an active, tight cooperation (compare [4], [12]) of slave robots coupled e.g. by a grasped object is considered. The presented teleoperation approach focuses on scenarios and systems as the walking robot setup of [13], the building of space structures by robots proposed by JAXA [12] and the Skyworker proposed in [14]. The focus of this paper lies on the cooperative telemanipulation of large or flexible objects by two human operators. A haptic intention augmentation is proposed that provides haptic cues to each operator on the desired direction of motion of the other operator observed by a force sensor at each input device. This solves the following challenges:

- When flexible objects are manipulated, the human operators A and B may coordinate the motions only via speech. Visual feedback to operator A provides 
just information on the position change of the slave hand controlled by human operator B as the applied force/acceleration of operator B is not directly visible. As measured force feedback from the slave interaction with a flexible object provides only blurred information, additional force feedback from the cooperating master interaction with its master device is crucial for a good cooperation performance.

- When large objects are manipulated, the inertia of the object and the inertia and damping of the teleoperation system influence the cooperation procedure. E.g., if a long object is grasped by one operator at each end, all intended motions apart from the one along the object may not be displayed optimally to the other operator. This holds true for the direct cooperation without a teleoperation system also. If operator A intends to move the object up, he has to apply a torque (acting with a lever arm against inertia and damping) at the same time in order to maintain the orientation of the object. In contrast, if only one person lifts an object with two hands she/he knows that both arms need to apply a force in the intended direction.

Among other things, these problems can be reduced by the feedforward of the interaction forces (and thus the intention of the respective operator) measured at the master device of operator A to operator B and vice versa. This can be regarded as a guidance of the cooperative operator with the second hand as depicted in Fig. 1 and Fig. 2. The control approach here is based on the 4-Channel setup presented in [15], [16]. A part of the experiments is conducted in the framework of the KONTUR-2 project [17].

The paper is structured as follows: Chapter II introduces one technical setup each for the cooperative manipulation of stiff and flexible objects. The control approach is presented in chapter III. Finally, chapter IV presents the experiment results and the conclusion is drawn in chapter V.

\section{TeChnical SetuP}

This chapter firstly presents the technical setup applied for the manipulation of stiff and secondly of flexible objects. The latter setup is finally validated in a space application.

\section{A. Stiff Object Manipulation}

The first setup considers the general case of interaction in a 6 degree of freedom (DoF) task space. The DLR SpaceJustin slave robot (see Fig. 3) is controlled via the DLR HUG haptic interface (see Fig. 4). Both systems are based on the DLR light weight robot technology (LWR).

Both slave robot hands grasp one stiff long object (pipe) at the two end positions. Two operators use one arm of HUG each as an input device. Fig. 2 presents the signal flow diagram for the haptic intention augmentation approach that is implemented for each DoF of the task space. The system can be divided into two 4-Channel teleoperation control loops (upper and lower half). The loops are connected via the delayed $\left(T_{3}\right)$ feedforward of the interaction force $f_{1 / 2, h}$ of the human operator $H_{1 / 2}$ with his/her master

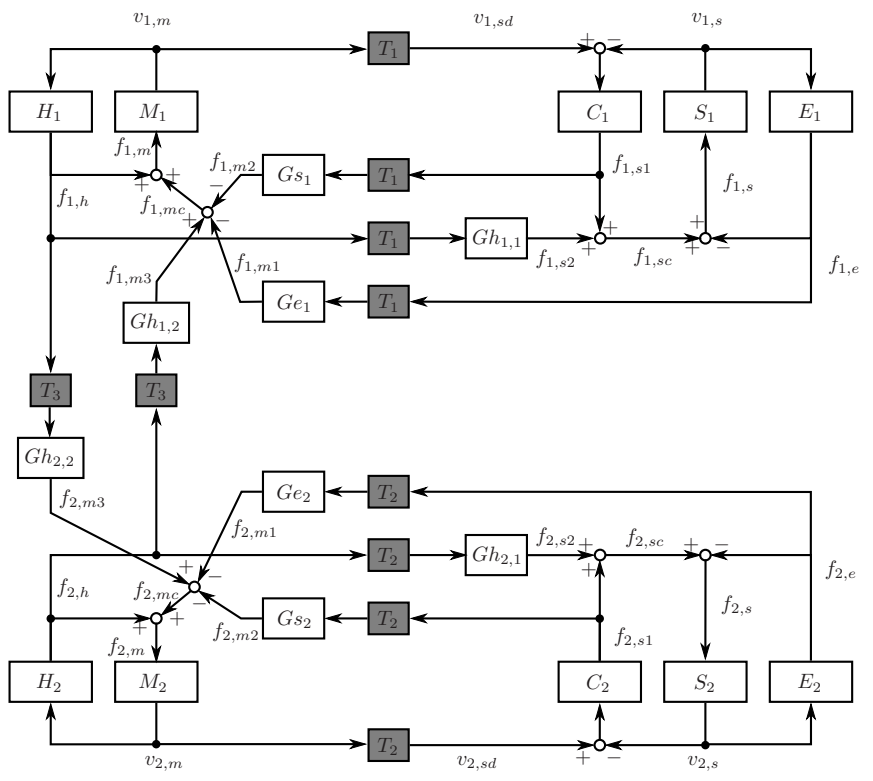

Fig. 2. Signal Flow Diagram of Haptic Intention Augmentation Setup

device $M_{1 / 2}$. These forces are scaled with the constant gains $G h_{1 / 2,2}$. In both teleoperator systems a time delay $T_{1 / 2}$ is considered in the communication channel. The forward and backward time delays are assumed to be equal for the sake of simplicity. Still, note that the chosen approach allows variable delay, jitter and package loss. A controller $C_{1 / 2}$ on the slave side $S_{1 / 2}$ aims at the position tracking of the master and slave robots. The admittance type slave receives in addition to the computed controller force, the scaled (gain $G h_{1 / 2,1}$ ) human interaction force. The master device $M_{1 / 2}$ receives the delayed computed controller force $f_{1 / 2, s 1}$ scaled by $G s_{1 / 2}$ and the measured environmental interaction force $f_{1 / 2, e}$ scaled by $G e_{1 / 2}$. With the $C_{1}$ controller impedance $Z_{c 1}(s)=\frac{K_{1, d s} s+K_{1, p s}}{s}$ the force command to slave 1 and master 1 result in:

$$
\begin{aligned}
f_{1, s c}(t)= & G h_{1,1} f_{1, h}\left(t-T_{1}\right)+K_{1, d s}\left(v_{1, m}\left(t-T_{1}\right)-\right. \\
& \left.v_{1, s}(t)\right)+K_{1, p s}\left(x_{1, m}\left(t-T_{1}\right)-x_{1, s}(t)\right), \\
f_{1, m c}(t)= & G s_{1} f_{1, s 1}\left(t-T_{1}\right)+G e_{1} f_{1, e}\left(t-T_{1}\right)+ \\
& G h_{1,2} f_{2, h}\left(t-T_{3}\right), \\
f_{1, s}(t)= & f_{1, s c}(t)-f_{1, e}, \\
f_{1, m}(t)= & f_{1, m c}(t)+f_{1, h} .
\end{aligned}
$$

\section{B. Flexible Object Manipulation}

The same control structure holds for the setup for the manipulation of flexible objects. The elasticity of the object reduces the quality of the perception of the cooperating operator's intention via the environment e.g. by measured force feedback. The two operators of the setup use a space qualified 2-DoF force feedback joystick (see Fig. 5) to control SpaceJustin. As the master devices have only two DoFs, the control of the 6-DoF humanoid robot arms had to be adapted. A sidewards and vertical motion of the arms could be commanded whereas the remaining four DoFs of the 


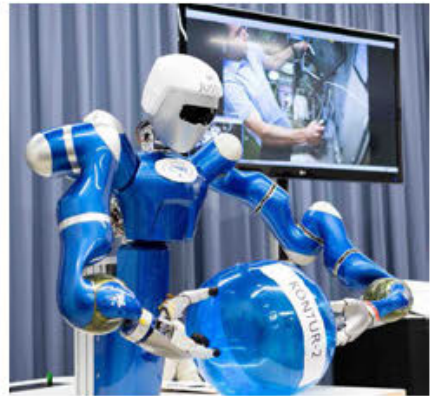

Fig. 3. DLR SpaceJustin with Flexible Object and Cosmonaut on ISS

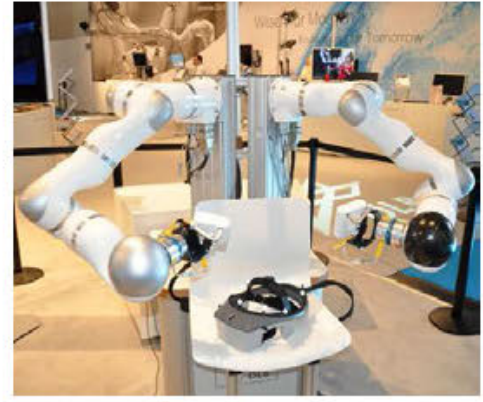

Fig. 4. DLR HUG Haptic Input Device

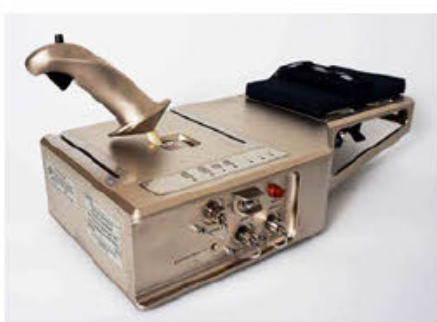

Fig. 5. DLR Kontur-2 Joystick robot were fixed to the initial position by a virtual springdamper system. Since the workspace of master and slave robot do not match, the indexing method was implemented to iteratively adapt the workspace of the master device.

In contrast to the experimental setup a flexible object had to be manipulated (compare Fig. 3). This object was not grasped by the hand but only held via opposing forces in horizontal direction from both slave robot arms. Furthermore, because of the permanent opposing horizontal forces that hold the object, the haptic intention augmentation approach was only activated in (vertical) z-direction.

\section{Stability Discussion}

The prevalent approaches aiming at the stabilization of teleoperation systems with respect to communication delay are the wave variables method [18] and the time domain passivity approach (TDPA, [19], [20]). Both methods are energy-based and consider the passivity-criterion, that guarantees $L_{2}$-stability:

$$
\int_{0}^{\infty} u(\tau) * v(\tau) d t+C \leq 0
$$

with the power correlated input signals of a dual port $u$ and $v$ and the initially $(t=0)$ stored energy C. The criterion implies that a system is passive as long as it does not generate energy. In order to analyze the energetic structure of a control loop, its electrical network representation based on the mechanical-electrical analogies is derived. Fig. 6 represents the upper half teleoperation scheme of the signal flow diagram of Fig. 2. The network representation of the haptic intention augmentation setup is based on the 4Channel architecture presented in [15] (similar to [21]). It includes the impedance of the human operator $Z_{1, h}$, the master device $Z_{1, m}$ and of the slave robot $Z_{1, s}$ in the environment $Z_{1, e}$. The operator's energy input is represented by the effort source $f 0_{1}$. In order to guarantee that the dual ports of all subsystems have a unique flow, the system is split up into two circuits that are connected with dependent effort and flow sources. The dependent effort sources $f_{1, m 1}, f_{1, m 2}$ and $f_{1, m 3}$ establish the feedback of the environmental force, the computed force of the controller $Z_{1, C}$ and the augmentation force of operator 2 . The dependent effort source $f_{1, s 1}$ provides the feedforward of the operator 1 interaction force.

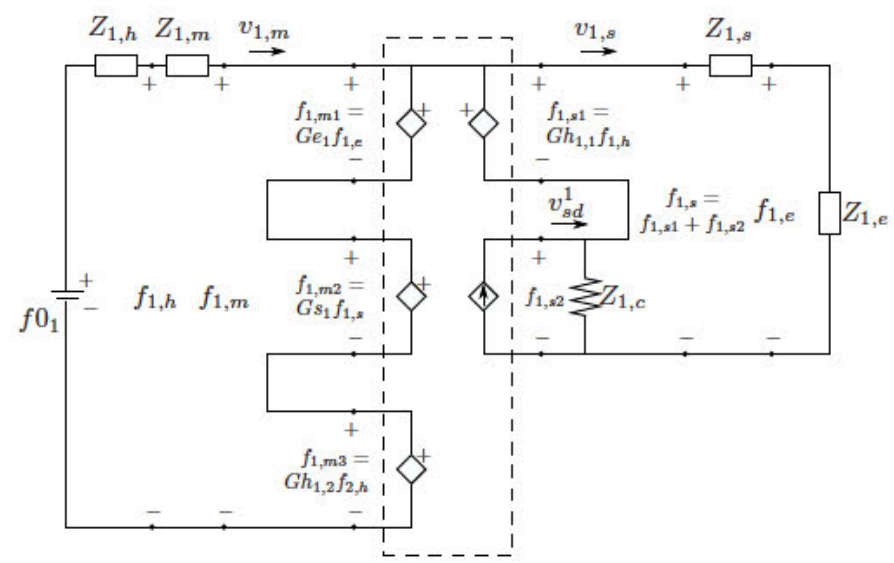

Fig. 6. One Half of the Network Representation of Haptic Intention Augmentation Setup without Time Delay

The dependent flow source $v_{1, s d}$ transmits the desired slave velocity to the slave side.

The system of Fig. 6 is passive if all subsystems are passive. Therefore, in the following, the effect of the time delay has to be considered, but also the passivity of the remaining subsystems has to be assured.

\section{A. Time Delay}

Since the 4-Channel architecture has one of the best performance with respect to transparency, the 4-Channel TDPA of [15] will be extended to the haptic intention augmentation approach in the following. The TDPA is applied to stabilize the teleoperation network despite delay, as it is robust to variable delay, jitter as well as package loss. Two passivity observers (PO) serve the measurement of the energy generated in a two-port subsystem and passivity controllers (PC) dissipate the excessive energy observed by the POs. An essential module of the TDPA is the two-port subsystem called Time Delay Power Network (TDPN, [22], [23]) that are introduced to assure the power consistency of transmitted signals in the communication channel. Via the TDPN energy flows that are not visible e.g. in the signal flow diagram become apparent such that it becomes obvious with which signals the POs have to be designed. Fig. 7 presents the network representation considering the TDPNs. The $T D P N_{1}$ A to D are similar to the design in [15]. 


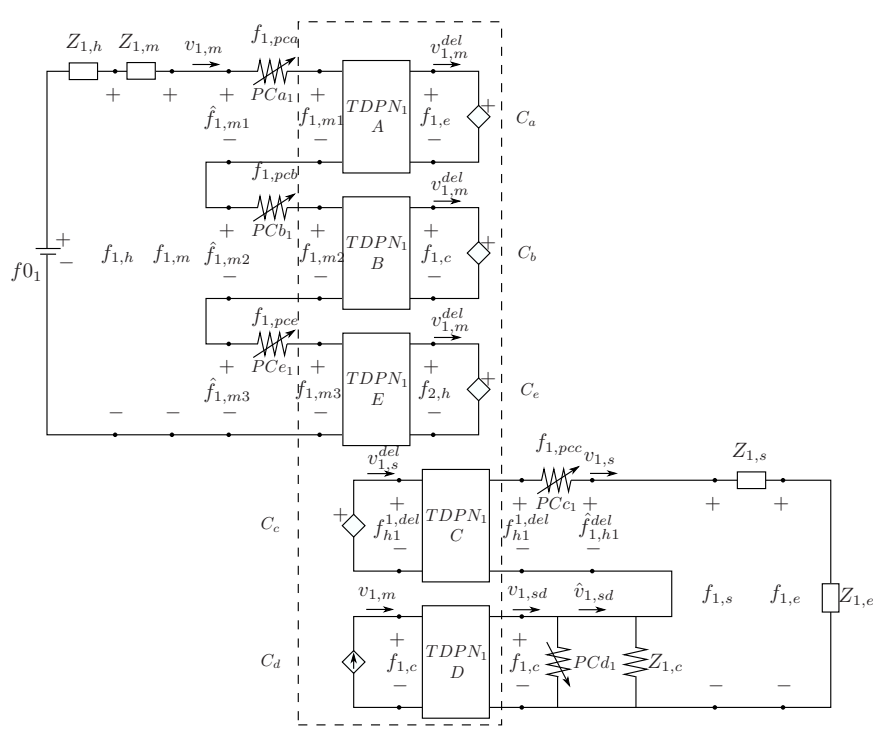

Fig. 7. Network Representation of Haptic Intention Augmentation Setup with Time Domain Passivity Control

The new haptic intention augmentation channel requires the introduction of the $T D P N_{1} \mathrm{E}$ with the conjugate pairs

$T D P N_{1}$ E: $\begin{cases}\left\langle G h_{1,2} f_{2, h}\left(t-T_{3}\right), v_{1, m}(t)\right\rangle & \text { at the } H_{1} \text { side } \\ \left\langle G h_{1,2} f_{2, h}(t), v_{1, m}\left(t-T_{3}\right)\right\rangle & \text { at the } H_{2} \text { side }\end{cases}$

The energy of the TDPN E $W_{1, M, e}$, observed by the PO, can be calculated in the discrete domain:

$$
W_{1, M, e}(n)=E_{1, \text { in }}^{H_{2}, e}(n-D)-E_{1, \text { out }}^{M_{1}, e}(n)+E_{1, P C}^{M_{1}, e}(n)
$$

with the discrete communication time delay $D$. The observed energies can be measured as follows:

- $E_{1, i n}^{H_{2}, e}(n-D)$ is the delayed energy entering (subscript in) the TDPN E from the human 2 side (superscript $\left.H_{2}, e\right)$, observed delayed at the master 1 side.

- $E_{1, \text { out }}^{M_{1}, e}(n)$ is the energy exiting (subscript out) the TDPN E at the human 1 side (superscript $M_{1}, e$ )

- $E_{1, P C}^{M_{1}, e}(n)$ is the energy dissipated by the passivity controller of TDPN E.

To calculate the observed energies, the direction of power flow has to considered:

$$
\begin{aligned}
& P_{1, \text { in }}^{H_{2}, e}(n)=\left\{\begin{array}{cc}
\tilde{f}_{2, h}(n)\left(-v_{1, m}\right. & \text { if } \tilde{f}_{2, h}(n)\left(v_{1, m}\right. \\
(n-D)) & (n-D))<0, \\
0 & \text { else }
\end{array}\right. \\
& P_{1, \text { out }}^{M_{1}, e}(n)=\left\{\begin{array}{cc}
\tilde{f}_{2, h}(n-D) & \text { if } \tilde{f}_{2, h}(n-D) \\
\left(-v_{1, m}(n)\right) & \left(v_{1, m}(n)\right)<0, \\
0 & \text { else }
\end{array}\right.
\end{aligned}
$$

with the scaled feedback force $\tilde{f}_{2, h}(n)=f_{2, h}(n) G h_{1,2}$. The observed energies result in:

$$
\begin{aligned}
& E_{1, \text { in }}^{H_{2}, e}(n)=E_{1, \text { in }}^{H_{2}, e}(n-1)+T_{s} P_{1, \text { in }}^{H_{2}, e}(n) \\
& E_{1, \text { out }}^{M_{1}, e}(n)=E_{1, \text { out }}^{M_{1}, e}(n-1)+T_{s} P_{1, \text { out }}^{M_{1}, e}(n),
\end{aligned}
$$

with the system sampling time $T_{s}$. The $P C e_{1}$ (see Fig. 7) dissipates the excessive energy $E_{1, P C}^{M_{1}, e}(n)$ in direction to the master. Concerning the functionality of the PC and the calculation of $E_{1, P C}^{M_{1}, e}(n)$ the reader is referred to [15].

\section{B. Overall Passivity}

Besides the passivity of the communication channel, the passivity of the other subsystems has to be proven. The controller as a spring damper system consists of passive modules. Also the master and slave systems, generally modeled as mass-damper systems, are passive. Concerning the human operators that are obviously introducing energy into the system, the following generally accepted assumption can be applied: Human operators and the slave environments behave passive in their interaction. This means that energy introduced by one will be dissipated by the other.

The additional feedforward of the operator interaction forces requires an additional analysis: If the cooperation would be performed without the teleoperation systems, the haptic intention augmentation approach is equal to the following scenario: The left hand of operator A applies a force according to the intended force on his right hand to the right hand of operator 2 in order to lead him into the intended direction (compare Fig. 1). In the real scenario this would not lead to instability and thus also the agents of the teleoperation setup will still behave passive in their interactions.

Furthermore, the effect of delay in general cooperative teleoperation setups [24] has to be analyzed. Since the delay $T_{3}$ is smaller than $T_{2}+T_{1}$, e.g. master 1 perceives the intention of master 2 earlier and better compared to the pure environmental feedback without haptic intention augmentation. Thus, the proper interaction of the operators is eased also in the delayed case.

Instability can only result from unintended energy generation. As discussed above, apart from the energy input of the human operators and the environment, no energy is generated in the system due to the passivity controllers. Therefore, the system stability can be guaranteed by the proper behavior of the human operators.

\section{RESUlTS}

This chapter presents at first the experiments with stiff and then with flexible object manipulation. The latter experiments also serve the analysis of the approach's capabilities in setups with time delay and bad quality of video feedback.

\section{A. Stiff Object Manipulation}

The delay in the 6-DoF experiments focusing stiff object manipulation was assumed to be zero. The slave robot's hands grasped a pipe firmly at a distance of about 0.6 meters. The feedback of haptic augmentation and environmental forces $f_{1 / 2, F B}$ are scaled in correlation with the scaling of the measured environmental feedback $\left(f_{1, F B}=G e_{1} f_{1, e, z}+\right.$ $\left.G h_{1,2} f_{2, h, z}, f_{2, F B}=G e_{2} f_{2, e, z}+G h_{1,2} f_{1, h, z}\right)$ such that the scaling $G e_{1 / 2}$ is reduced when $G h_{1 / 2,2}$ is increased. $G e_{1 / 2}$ was 0.8 and $G h_{1 / 2,2}$ had the value 0.6.

Experiments Exp1.1a and Exp1.1b focus the functionality of the haptic intention augmentation in the translational degrees of freedom. The left operator 1 starts moving the left slave hand upwards (10.5s, Fig. 8). The right operator 2 follows this motion in order to keep the pipe horizontal as soon as he has perceived the intention of operator 1 . Without 

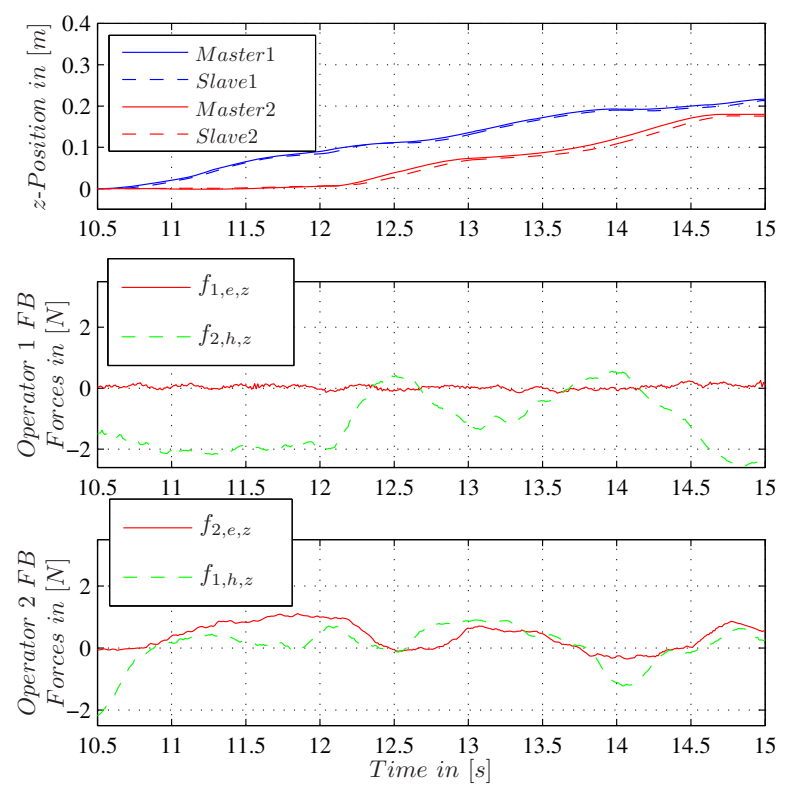

Fig. 8. Exp1.1a: Cooperative Motion in z-Direction without Haptic Intention Augmentation

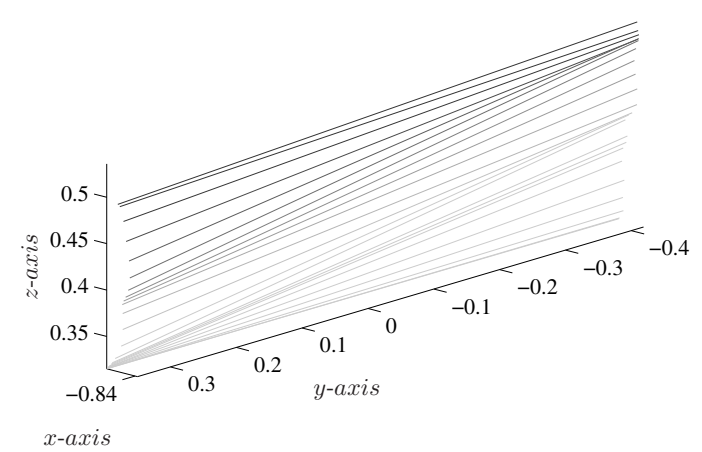

Fig. 9. Exp1.1a: Translational 3D Pipe Motion without Haptic Intention Augmentation

intention augmentation (Exp1.1a) the operator relies on the visual feedback of the slave motion only. In experiment Exp1.1b with active haptic intention augmentation the right operator 2 receives the sum of the left operator's interaction force $f_{1, h}$ and the much lower measured force feedback $f_{2, e}$. Comparing Fig. 8 and Fig. 10 it is obvious that the operator 2 starts following the translational motion earlier with active augmentation. Also the average distance in $\mathrm{z}$ direction is lower for that case. The 3D pipe motion during this procedure is visualized in Fig. 9 and Fig. 11. The line color in the 3D-Plot implies the time. With increasing time, the color gets darker. It is obvious that the pipe motion is less horizontal without haptic augmentation.

The second experiment Exp1.2 analyzes the benefit of the haptic intention augmentation applied during rotational motions. The left operator 1 intends to rotate the pipe around the right slave robot hand. Analogous to the forces, the environmental interaction torques around the $\mathrm{x}$-axis $\tau_{2, e, \alpha}$ are in both experiments (Exp1.2a in Fig. 12 and Exp1.2b in Fig.
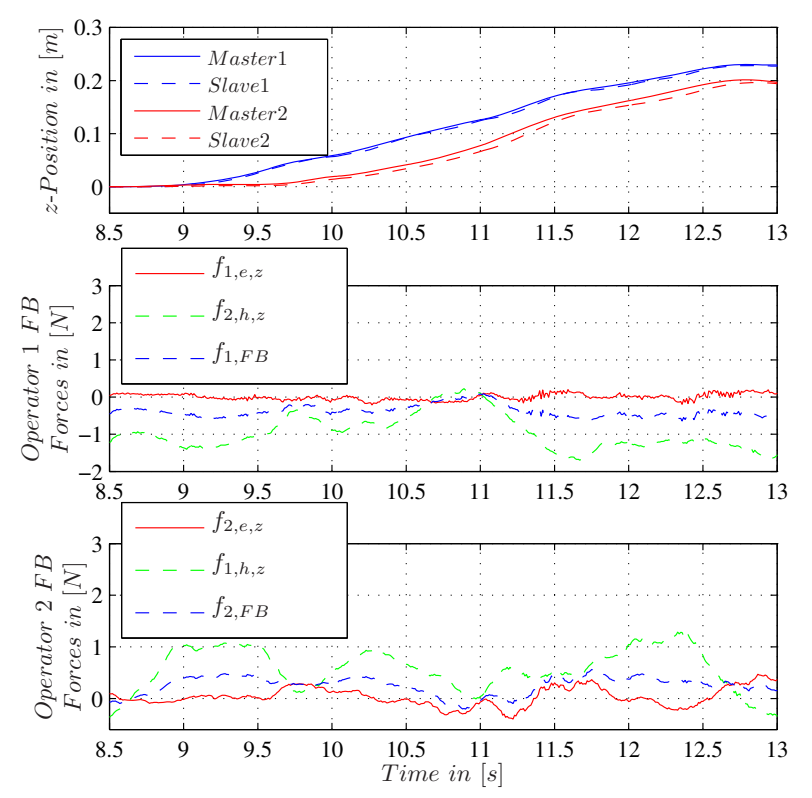

Fig. 10. Exp1.1b: Cooperative Motion in z-Direction with Haptic Intention Augmentation

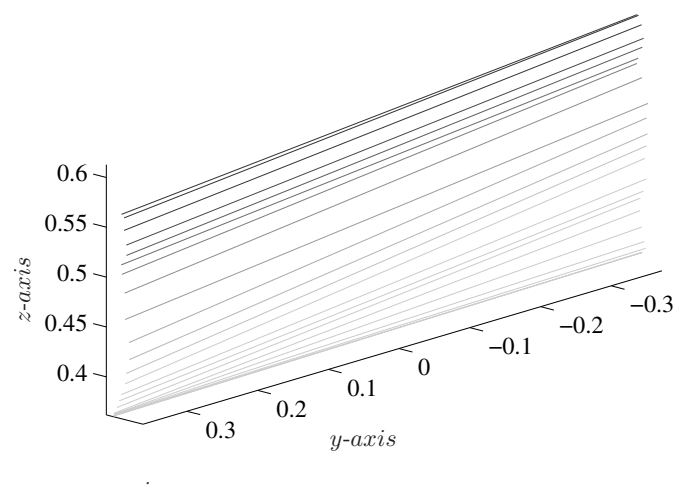

$x$-axis

Fig. 11. Exp1.1b: Translational 3D Pipe Motion with Haptic Intention Augmentation

13) much lower than the operator's interaction torque $\tau_{1, h, \alpha}$ with the human machine interface. Without the augmentation concept, the operators obviously receive less haptic cues to react on the other operator's motion. Comparing Fig. 12 and Fig. 13, operator 2 reacts earlier on the change in rotation and the average of difference in angle $\alpha$ is lower in Exp1.2b with haptic intention augmentation though the time of completion is much shorter.

\section{B. Flexible Object Manipulation}

The following experiment Exp2 refers to the ISS communication setup presented in Fig. 14. A cosmonaut on the ISS, along with an operator in Russia, controls the two arms of the humanoid robot Justin located in Germany. The communication from Oberpfaffenhofen (Germany) to Saint-Petersburg was a standard internet connection $(70 \mathrm{~ms}$ roundtrip delay). The link to ISS included a high-speed internet link from Oberpfaffenhofen to Weilheim (Germany) 

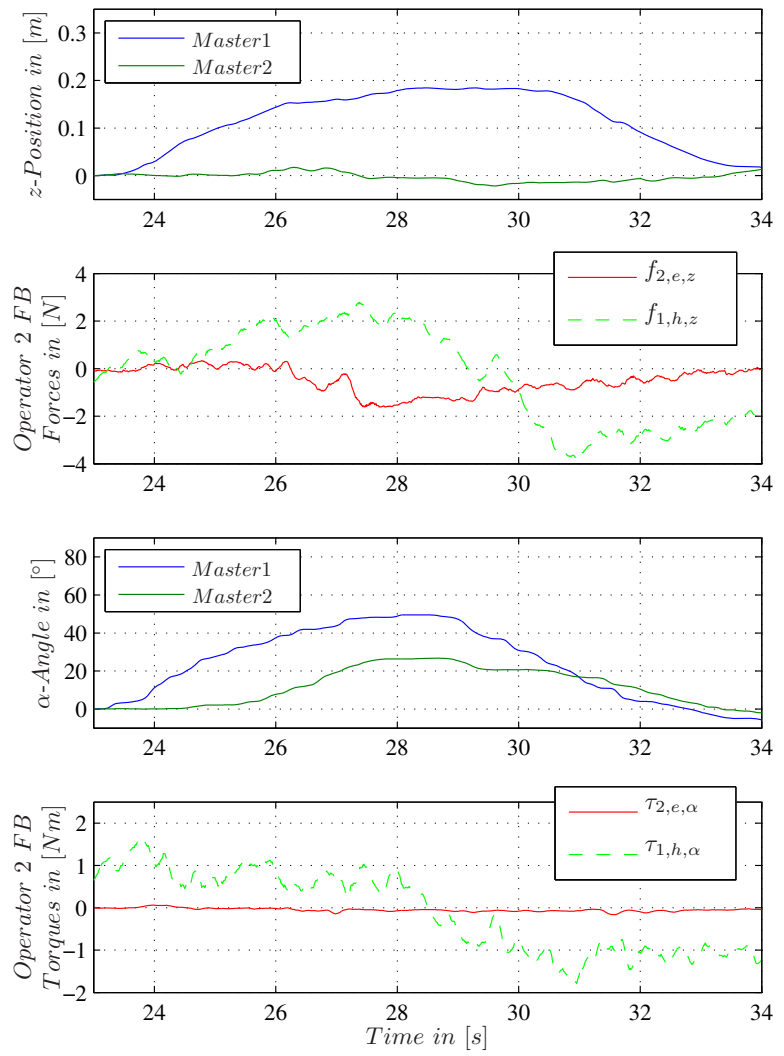

Fig. 12. Exp1.2a: Operator 1 Force and Torque Feedback during Rotation around Right Slave Robot Hand without Haptic Intention Augmentation

and a S-band communication to the ISS (25ms roundtrip delay). The communication was affected by delay, jitter and package loss. The visual feedback was of low quality due to the available bandwidth.

At first, the operators had to approach the ball from the side and hold it with opposing forces. The motion had to be performed as simultaneously as possible in order to hold the ball. The ball had to be moved upwards (z-axis) and right (x-axis) with haptic intention augmentation (compare Fig. 15). $G e_{1 / 2}$ was 0.3 and $G h_{1 / 2,2}$ had the value 0.6 . As it can be seen in Fig. 16, the measured environmental interaction force $f_{1, e, z}$ is in absolute value smaller than the operator 2 interaction force $f_{2, h, z}$. The elasticity of the object as well as the damping and the mass of master and slave devices blur the intention of the cooperating operators.

As it can be seen in Fig. 16, the haptic intention augmentation (forces $f_{1 / 2, F B}$ ) enabled the transmission of more interaction information. The disturbances in the motion result from robot workspace nonlinearities and the elasticity of the manipulated object. Despite that and the high distance in zdirection between the grasping positions, it can be seen in Fig. 15 that the cooperation procedure was successful.

\section{CONCLUSION}

The main contributions of this paper are the development of a novel cooperation architecture and the validation of the proposed approach in a setup involving microgravity
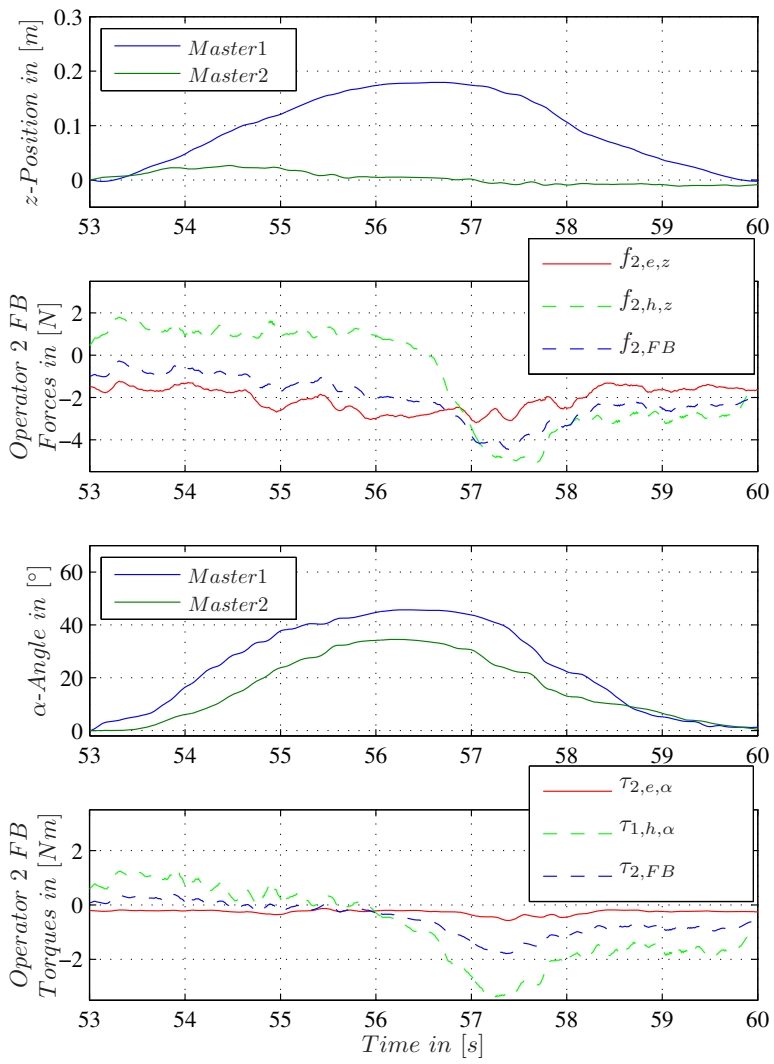

Fig. 13. Exp1.2b: Operator 1 Force and Torque Feedback during Rotation around Right Slave Robot Hand with Haptic Intention Augmentation

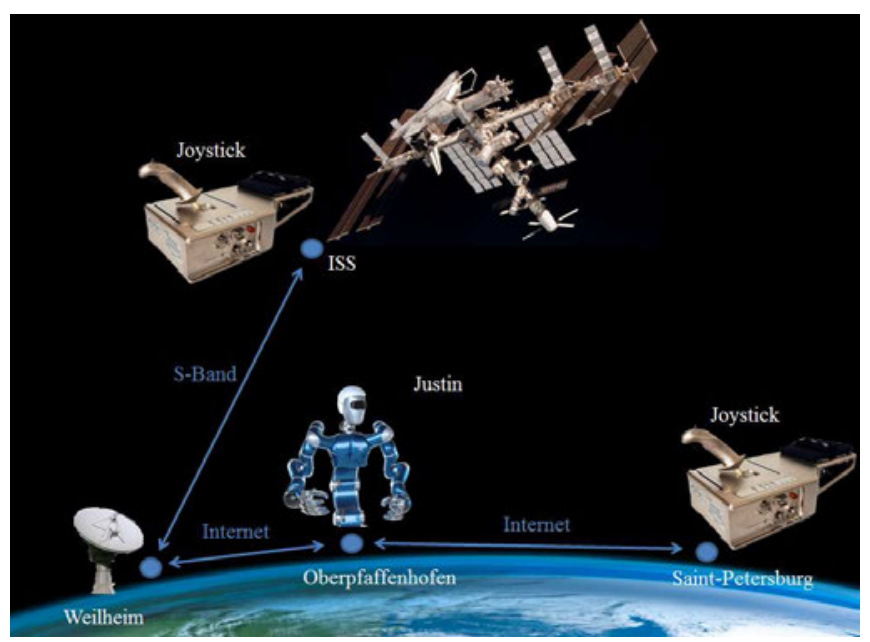

Fig. 14. Communication Infrastructure 


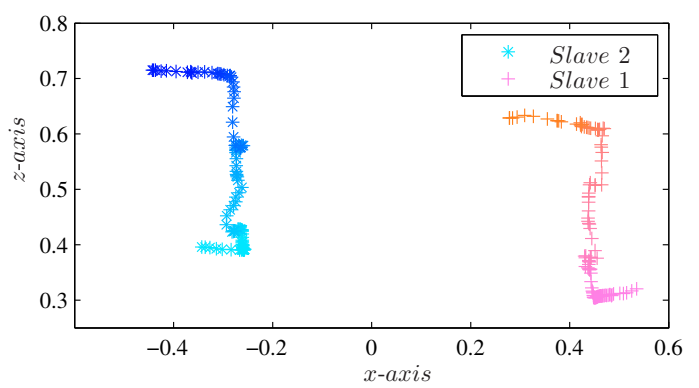

Fig. 15. Exp2.2: 2D Position Tracking with Haptic Intention Augmentation
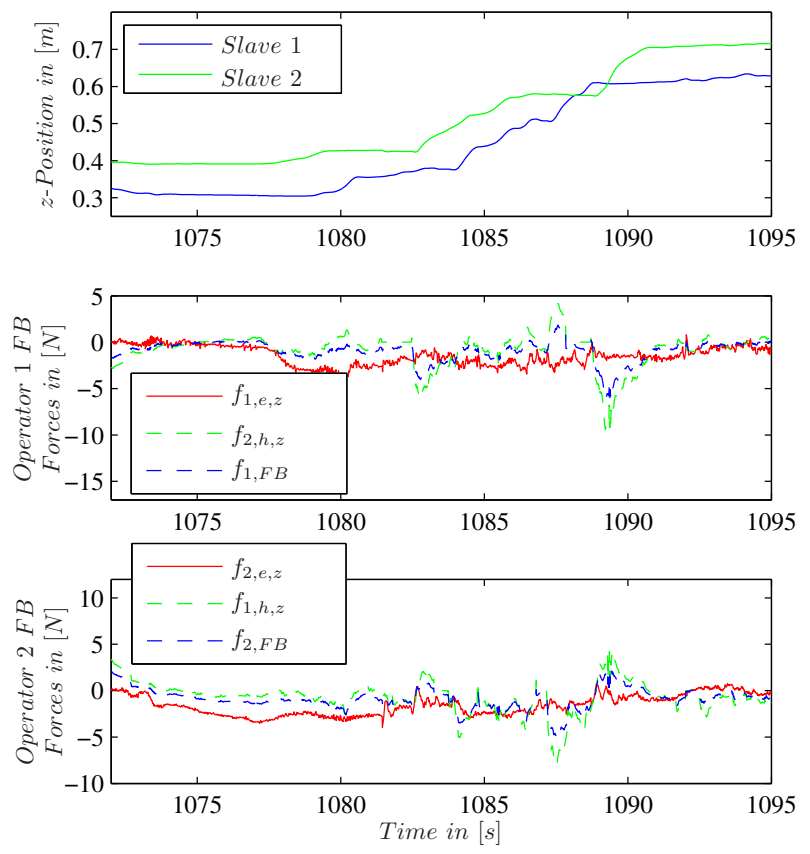

Fig. 16. Exp2.2: Interaction Forces with Haptic Intention Augmentation

conditions and real space communication links. A haptic intention augmentation has been proposed that is suitable for the handling of long and/or flexible objects in a tight cooperation manner. Despite the low quality of the visual feedback, the experiments in the space setup showed satisfactory results. The cooperation setup showed to be robust to communication channels, affected by high time delay, jitter and high package loss. In future work, the generalization and adaptability of the intention augmentation approach to other tasks should be further analyzed.

\section{ACKNOWLEDGMENT}

The authors would like to thank the project partners ROSKOSMOS, RSC Energia, RTC and the cosmonauts Oleg Kononenko and Sergej Volkov. Furthermore, the authors want to express their sincere gratitude towards Martin Stelzer and Peter Birkenkampf (DLR-RM) for their unconditional and essential technical support.

\section{REFERENCES}

[1] W. W. Mendell, "The roles of humans and robots in exploring the solar system," Acta astronautica, vol. 55, no. 2, pp. 149-155, 2004.

[2] J. William and F. Ballhaus, "James Webb Space Telescope, Independent Comprehensive Review Panel, Final Report," NASA contractor report, Scientific and Technical Inf. Branch, vol. 3, 2010.

[3] S. Sirouspour and P. Setoodeh, "Multi-operator/multi-robot teleoperation: an adaptive nonlinear control approach," in Int. Conf. on Intelligent Robots and Systems, 2005, pp. 1576-1581.

[4] J. Leitner, "Multi-robot cooperation in space: A survey," in Advanced Technologies for Enhanced Quality of Life, 2009, pp. 144-151.

[5] N. Y. Chong, T. Kotoku, K. Ohba, and K. Komoriya, "Remote Coordinated Controls in Multiple Telerobot Cooperation," Int. Conf. on Robotics and Automation, pp. 3138 - 3143, 2000.

[6] J. Ferketic, L. Goldblatt, E. Hodgson, S. Murray, R. Wichowski, A. Bradley, T. Fong, J. Evans, W. Chun, R. Stiles et al., "Toward human-robot interface standards II: An examination of common elements in human-robot interaction across the space enterprise," in AIAA Space, 2006.

[7] T. Fong and I. Nourbakhsh, "Interaction challenges in human-robot space exploration," NASA technical report, 2005.

[8] Passenberg, Carolina and Peer, Angelika and Buss, Martin, "Modelmediated teleoperation for multi-operator multi-robot systems," Int. Conf. on Intelligent Robots and Systems, pp. 4263 - 4268, 2010.

[9] S. Sirouspour, "Robust control design for cooperative teleoperation," in Int. Conf. on Robotics and Automation, 2005, pp. 1133-1138.

[10] I. Elhajj, N. Xi, W. Fung, Y.-H. Liu, Y. Hasegawa, and T. Fukuda, "Modeling and control of internet based cooperative teleoperation," in Int. Conf. on Robotics and Automation, vol. 1, 2001, pp. 662-667.

[11] W.-t. Lo, Y. Liu, I. H. Elhajj, N. Xi, Y. Wang, and T. Fukuda, "Cooperative teleoperation of a multirobot system with force reflection via internet," Trans. on Mechatronics, vol. 9, no. 4, pp. 661-670, 2004.

[12] Y. Ishijima, D. Tzeranis, and S. Dubowsky, "The on-orbit maneuvering of large space flexible structures by free-flying robots," in Proc. of $i$ SAIRAS Conf., 2005.

[13] M. Oda and M. Mori, "Stepwise development of SSPS; JAXAs current study status of the $1 \mathrm{GW}$ class operational SSPS and its precursor," Int. Astronautical Congress, vol. 1, no. 10, 2003.

[14] P. J. Staritz, S. Skaff, C. Urmson, and W. Whittaker, "Skyworker: a robot for assembly, inspection and maintenance of large scale orbital facilities," in Int. Conf. on Robotics and Automation, vol. 4, 2001, pp. $4180-4185$.

[15] J. Artigas, R. Balachandran, C. Riecke, M. Stelzer, B. Weber, J.H. Ryu, and A. Albu-Schaeffer, "KONTUR-2: force-feedback teleoperation from the Int. space station," in Int. Conf. on Robotics and Automation, 2016, pp. 1166-1173.

[16] D. A. Lawrence, "Stability and transparency in bilateral teleoperation," Trans. on Robotics and Automation, vol. 9, no. 5, pp. 624-637, 1993.

[17] C. Riecke, J. Artigas, R. Balachandran, R. Bayer, A. Beyer, B. Brunner, H. Buchner, T. Gumpert, R. Gruber, F. Hacker, K. Landzettel, G. Plank, S. Schtzle, H.-J. Sedlmayr, N. Seitz, B.-M. Steinmetz, M. Stelzer, J. Vogel, B. Weber, B. Willberg, and A. Albu-Schffer, "Kontur-2 mission: the dlr force feedback joystick for space telemanipulation from the iss," in Proc. of i-SAIRAS Conf., 2016.

[18] G. D. Niemeyer, "Using wave variables in time delayed force reflecting teleoperation," Ph.D. dissertation, Massachusetts Institute of Technology, 1996.

[19] J.-H. Ryu, D.-S. Kwon, and B. Hannaford, "Stable teleoperation with time-domain passivity control," Trans. on Robotics and Automation, vol. 20, no. 2, pp. 365-373, 2004.

[20] J.-H. Ryu, J. Artigas, and C. Preusche, "A passive bilateral control scheme for a teleoperator with time-varying communication delay," Mechatronics, vol. 20, no. 7, pp. 812-823, 2010.

[21] J. Rebelo and A. Schiele, "Time domain passivity controller for 4channel time-delay bilateral teleoperation," Trans. on Haptics, vol. 8 , no. 1, pp. 79-89, 2015

[22] J. Artigas, J.-H. Ryu, C. Preusche, and G. Hirzinger, "Network representation and passivity of delayed teleoperation systems," in Int. Conf. on Intelligent Robots and Systems, 2011, pp. 177-183.

[23] J. Artigas Esclusa, "Time domain passivity control for delayed teleoperation," Ph.D. dissertation, 2014.

[24] S. Sirouspour and P. Setoodeh, "Adaptive nonlinear teleoperation control in multi-master/multi-slave environments," in Control Applications, 2005. CCA 2005. Proc. of 2005 IEEE Conf. on. IEEE, 2005, pp. 1263-1268. 